\title{
Color Stability of Acrylic Resin Adhesives with Different Initiation Modes
}

\author{
Naomi TANOUE ${ }^{1}$, Yoshikazu KOISHI ${ }^{2}$, Hiroaki YANAGIDA ${ }^{3}$, Mitsuru ATSUTA $^{4}$, Kazuki SHIMADA ${ }^{5}$ \\ and Hideo MATSUMURA ${ }^{5}$ \\ ${ }^{1}$ Department of Specialized Dentistry, Nagasaki University Hospital of Medicine and Dentistry, 1-7-1, Sakamoto, Nagasaki \\ 852-8588, Japan \\ ${ }^{2}$ Department of Dental Materials, Nihon University School of Dentistry at Matsudo, 2-870-1, Sakaecho Nishi, Matsudo, Chiba \\ 271-8587, Japan \\ ${ }^{3}$ Department of General Dentistry, Nagasaki University Hospital of Medicine and Dentistry, 1-7-1, Sakamoto, Nagasaki 852- \\ 8588, Japan \\ ${ }^{4}$ Division of Fixed Prosthodontics and Oral Rehabilitation, Nagasaki University Graduate School of Biomedical Sciences, 1-7- \\ 1, Sakamoto, Nagasaki 852-8588, Japan \\ ${ }^{5}$ Department of Crown and Bridge Prosthodontics, Nihon University School of Dentistry, 1-8-13, Kanda-Surugadai, Chiyoda- \\ ku, Tokyo 101-8310, Japan \\ Corresponding author, E-mail:t-naomi@net.nagasaki-u.ac.jp
}

Received April 14, 2004/Accepted June 24, 2004

\begin{abstract}
The purpose of this study was to evaluate the color stability of two acrylic resin adhesives with different activation systems: a benzoyl peroxide (BPO)-amine redox system and a tri- $n$-butylborane (TBB) derivative system. The colorimetric values of the two resins in different shades (Clear and Ivory) were determined $(n=5) 24$ hours after polymerization as a baseline using the $\mathrm{L}^{*} \mathrm{a}^{*} \mathrm{~b}^{*}$ system of the Commission Internationale de l'Eclairage (CIE). The specimens were thereafter immersed in distilled water, and the color difference $\left(\Delta \mathrm{E}^{*}\right)$ values were calculated. After 24 weeks, the TBB-initiated material showed a significantly $(p<0.05)$ lower color change than the BPO-amine-initiated material. The $\Delta \mathrm{E}^{*}$ values for the BPO-amineinitiated materials were 6.9 for Clear and 15.8 for Ivory, whereas those for the TBB-initiated materials were 1.3 and 1.8 respectively. Thus, it was concluded that the TBB-initiated material had superior color stability to that of the BPO-amineinitiated material.
\end{abstract}

Key words: Acrylic resin, Adhesive, Amine, Color, Initiator, 4-META, Tri-n-butylborane

\section{INTRODUCTION}

The mechanical and handling properties of resinbased luting agents have been considerably improved during the last decade, and these reliable properties have led to the materials being selected for cementation as well as color adjustment of varying restorations ${ }^{1)}$. In addition, mainly due to the satisfactory mechanical properties and bond strengths to teeth, the agents have also been used as resin splints for stabilization of mobile anterior teeth without wire $^{2,3)}$. The acrylic resin adhesives can therefore be applied for various purposes in general dental practice.

Currently, there are a number of commercially available adhesives, and the polymerization systems are also various and characteristic. Super-Bond C\&B (SB; Sun Medical Co. Ltd., Moriyama, Japan) is a representative chemically-cured adhesive, which due to its strong bonding to the tooth substrate is widely used for bonding restorations and fixed-partial dentures as well as splints ${ }^{4)}$. Multi-Bond (MB; Tokuyama Dental Corp., Tokyo, Japan), which is developed recently, is another type of chemically-cured acrylic adhesive ${ }^{5}$. Although both materials are based on methyl methacrylate (MMA), the SB material is initiated by tri- $n$-butylborane (TBB), while the MB material is initiated by a benzoyl peroxide (BPO)amine redox system.

In the case of splints for mobile anterior teeth and/or cementation of tooth-colored restorations including both porcelain and indirect resin composites, the adhesives are required to have sufficient color stability for esthetic reasons. Color stability of dualpolymerized composites is affected by either the intensity of the light source or the light-exposure time period applied to the materials ${ }^{6}$. The color of acrylic materials based on MMA has been reported to change over long-term use ${ }^{7-9)}$. Unlike dual- or lightpolymerizing systems, the majority of MMA-based adhesive resins contain either amine reducing agent or acidic functional monomer, or both, in the MMA liquid component. If a material contains both the amine reducing agent and the acidic functional monomer in the liquid component, either the polymerization reaction or the color stability is negatively affected by formation of an acid-base charge transfer complex. Although the manufacturer of this currently available product (i.e., the MB material) claims that no problem has been highlighted regarding the addition of an acidic compound together with an amine reducing agent to the MMA liquid, limited 
information is available about the post-curing properties of the MB material, especially in respect to its long-term color stability. Contrarily, the TBB initiator for the SB material is stored in a syringe, which is separated from the monomer liquid. In addition, the TBB system does not use an amine reducing agent.

The purpose of this study, therefore, was to quantitatively evaluate the discoloration of two noted acrylic resin adhesives, each of which contains an acidic functional monomer. The two materials were intentionally selected because of their different initiation systems.

\section{MATERIALS AND METHODS}

Two acrylic adhesives (MB and SB) in different shades (Clear and Ivory) were selected. The MB material consisted of two components (liquid and powder), whereas the SB material was composed of three components (liquid, powder, and initiator). Both materials contained poly-MMA (PMMA) in the powder and MMA in the liquid. The MB material contained 11-methacryloyloxyl undecan-1,1-dicarboxlic acid (MAC-10) in the MMA monomer liquid, while the SB material contained 4-methacryloyloxyethyl trimellitate anhydride (4-META) as the adhesivepromoting monomer. The $\mathrm{MB}$ adhesive specifically included BPO in the powder and an amine reducing agent in the liquid. Information pertaining to the acrylic adhesives assessed is summarized in Table 1.

Stainless steel molds (inside diameter $6.0 \mathrm{~mm}$ and thickness $1.0 \mathrm{~mm}^{10,11)}$ ) on a glass plate were filled with acrylic adhesive material of each shade and then covered by another glass plate. The molds were then pressed between the glass plates at room temperature for 30 minutes. All materials were used in accordance with the manufacturers' specifications. The specimens were removed from the molds and stored in an incubator at $37^{\circ} \mathrm{C}$ and $100 \%$ humidity for 24 hours. For each material and shade, five specimens were subjected to color evaluation.

After 24 hours, baseline color measurements were performed as described below. The specimens were stored in distilled water at $37^{\circ} \mathrm{C}$ for up to 24 weeks. Color was evaluated using a small-area $(3 \mathrm{~mm}$ in diameter) dental chroma meter (ShadeEye, Shofu Inc., Kyoto, Japan) connected to a personal computer (FMV-Biblo NUVI23D, Fujitsu, Tokyo, Japan). The color parameters were expressed using the Commission Internationale de l'Eclairage (CIE) illuminant D65 observer functions ( $2^{\circ}$ visual field) and tabulated into the CIE $\mathrm{L}^{*} \mathrm{a}^{*} \mathrm{~b}^{*}$ coordinates for each material, using a software package for colorimetric analysis (Saicheck for Windows 98, IPA, Tokyo, Japan). The chroma meter was calibrated at the start of each specimen measurement using the white calibration cap supplied by the manufacturer. For all color measurements, the tip of the dental chroma meter was positioned perpendicularly on the surface of the specimen backed by mid-gray cardboard (Color Tile CM-A 101 MG, Minolta, Osaka, Japan), and readings were taken at five arbitrarily selected points on the top surface of the specimen. The mid-gray cardboard was used to eliminate as much as possible any background effect ${ }^{12,13)}$.

The $L^{*} a^{*} b^{*}$ values were averaged to obtain a single set of values for each specimen, and the mean and standard deviations (s.d.) of the five replications were calculated. The $L^{*}$ value is called the phychometric lightness, and the higher the $\mathrm{L}^{*}$ value the higher the lightness. The $\mathrm{a}^{*} \mathrm{~b}^{*}$ values are the phychometric chroma coordinates, or chromaticity. The $\mathrm{a}^{*}$ value indicates the red color on the plus side and the green color on the minus side, while the $\mathrm{b}^{*}$ value shows the yellow color on the plus side and the blue color on the minus side. The higher the values, the stronger the color factors are.

The color difference $\left(\Delta \mathrm{E}^{*}\right)$ values between 24 hours and other immersion periods $(1,2,3,4,8,12$, 16,20 , and 24 weeks) were calculated for each specimen as follows:

$$
\Delta \mathrm{E}^{*}=\left[\left(\Delta \mathrm{L}^{*}\right)^{2}+\left(\Delta \mathrm{a}^{*}\right)^{2}+\left(\Delta \mathrm{b}^{*}\right)^{2}\right]^{1 / 2}
$$

where $\Delta \mathrm{L}^{*}, \Delta \mathrm{a}^{*}$, and $\Delta \mathrm{b}^{*}$ are the differences in the respective $\mathrm{L}^{*}, \mathrm{a}^{*}$, and $\mathrm{b}^{*}$ values.

The mean and s.d. of the $\Delta \mathrm{E}^{*}$ values of each specimen were then calculated and analyzed by re-

Table 1 Details of the acrylic adhesives assessed

\begin{tabular}{|c|c|c|}
\hline Material/Abbreviation & Multi-Bond/MB & Super-Bond/SB \\
\hline $\begin{array}{l}\text { Powder/Shade } \\
\text { (Batch number) }\end{array}$ & $\begin{array}{cc}\text { PMMA, BPO/Clear } & \text { PMMA, } \begin{array}{l}\text { BPO, Pigments/Clear } \\
\text { (DMBPC-501) }\end{array} \\
\text { (DMPBI-401) }\end{array}$ & $\begin{array}{cc}\text { PMMA/Clear } \\
\text { (VE1) }\end{array}$ \\
\hline $\begin{array}{l}\text { Liquid } \\
\text { (Batch number) }\end{array}$ & $\begin{array}{l}\text { MMA, MAC-10, Multi-function monomer, Amine } \\
\text { (DMBML-3) }\end{array}$ & $\begin{array}{l}\text { MMA, 4-META } \\
\text { (EF1) }\end{array}$ \\
\hline $\begin{array}{c}\text { Initiator } \\
\text { (Batch number) }\end{array}$ & & $\begin{array}{l}\text { TBB } \\
(\text { VW61) }\end{array}$ \\
\hline Manufacturer & Tokuyama Dental Corp., Tokyo, Japan & Sun Medical Co., Moriyama, Japan \\
\hline
\end{tabular}


peated-measure analysis of variance (ANOVA) to investigate the influences of the material, shade, and immersion period. In addition, the $\Delta \mathrm{E}^{*}$ values after 24 weeks were compared by two-way ANOVA, oneway ANOVA, and Tukey-Kramer multiple comparison intervals, with the value of statistical significance set at $\mathrm{p}=0.05$.

\section{RESULTS}

Repeated-measure ANOVA performed on the $\Delta \mathrm{E}^{*}$ values indicated that the interactions among the materials, shade and immersion periods, and the interactions between each pair of two factors were significantly different $(p<0.05)$. The $\Delta E^{*}$ values were also affected by material, shade, and immersion period.

Changes in the $\Delta \mathrm{E}^{*}$ values are shown in Fig. 1 . The $\Delta \mathrm{E}^{*}$ values of the MB materials increased to a greater extent than those of the SB materials with the immersion period. The two-way ANOVA run on the $\Delta \mathrm{E}^{*}$ values after 24 weeks revealed that the interaction between the material and the shade $(\mathrm{F}=35.1, \mathrm{p}=0.0001)$ was significant and that color change was affected by both material type $(\mathrm{F}=187.3$, $\mathrm{p}=0.0001)$ and shade $(\mathrm{F}=42.6, \mathrm{p}=0.0001)$. The $\Delta \mathrm{E}^{*}$ values were therefore analyzed by one-way ANOVA

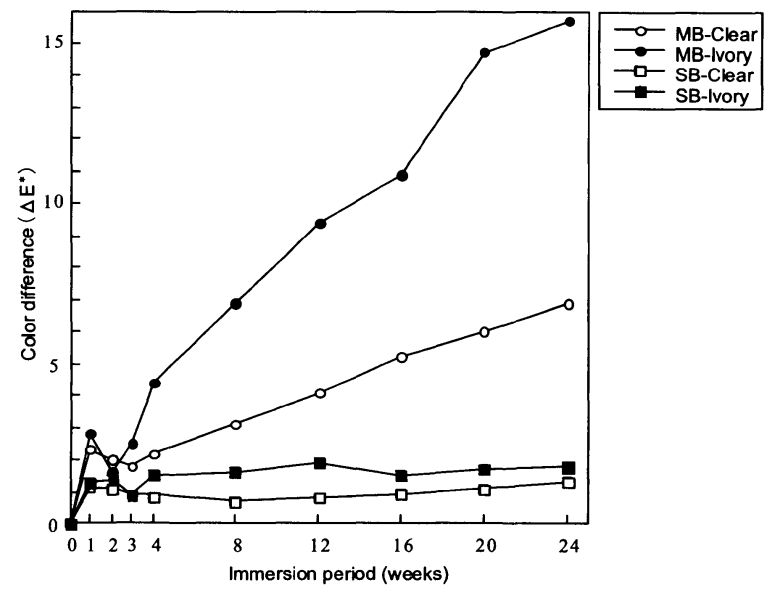

Fig. 1 Changes in the $\Delta E^{*}$ values for up to 24 weeks for each acrylic adhesive. and post-hoc Tukey-Kramer multiple comparison intervals. The results for the $\Delta \mathrm{L}^{*}, \Delta \mathrm{a}^{*}, \Delta \mathrm{b}^{*}$, and $\Delta \mathrm{E}^{*}$ values after 24 weeks and the Tukey-Kramer intervals are presented in Table 2. Regarding the MB material, the $\Delta b^{*}$ values changed more notably than the $\Delta \mathrm{L}^{*}$ and $\Delta \mathrm{a}^{*}$ values, whereas the SB material indicated little color change for all values. Irrespective of the shade, the $\Delta \mathrm{E}^{*}$ values for the $\mathrm{SB}$ material after 24 weeks were significantly $(p<0.05)$ smaller than those for the MB material. Although there was a significant difference $(p<0.05)$ between the $\Delta \mathrm{E}^{*}$ values for the MB-Clear and MB-Ivory materials after 24 weeks, there were no significant differences $(p>0.05)$ between the SB-Clear and SB-Ivory materials.

\section{DISCUSSION}

The color changes that occurred in the resin adhesives assessed in the current study were proved to be influenced by three factors, i.e., the type of material, shade, and immersion period. Based on previous reports ${ }^{10,11}$, the current study used $1-\mathrm{mm}$ thick specimens in consideration of the fact that the luting agents are applied intraorally with a thin layer of less than $0.5 \mathrm{~mm}$, whereas the adhesives for provisional splinting are used with a thickness of more than $1 \mathrm{~mm}$, especially around the proximal contact areas.

It was reported that discoloration of resin-based materials was caused by water immersion ${ }^{14-17)}$. Water has an important role in the chemical degradation of resin materials, resulting in both hydrolytic reactions and material swelling ${ }^{18)}$. The degree of degradation depends on the chemical composition of the monomer, the degree of cross-linking in the polymerized matrix, and the degree of conversion ${ }^{19)}$. For the reasons mentioned above, except for crosslinking, the adhesives assessed are supposed to have changed their colors gradually.

Regarding the influence of material type, differences in the compositions between the two adhesives might be related to their color stability. The MB material indicated significant color changes in comparison with the SB material. As shown in Table 1, the liquid of the $\mathrm{MB}$ material contains an amine reducing agent. Asmussen ${ }^{20)}$ reported that discolora-

Table $2 \Delta \mathrm{L}^{*}, \Delta \mathrm{a}^{*}, \Delta \mathrm{b}^{*}$, and $\Delta \mathrm{E}^{*}$ values for each acrylic adhesive after 24 weeks

\begin{tabular}{crrrrc}
\hline Material & \multicolumn{1}{c}{$\Delta \mathrm{L}^{*}$} & \multicolumn{1}{c}{$\Delta \mathrm{a}^{*}$} & \multicolumn{1}{c}{$\Delta \mathrm{b}^{*}$} & \multicolumn{1}{c}{$\Delta \mathrm{E}^{*}$} & $\Delta \mathrm{E}^{*}$ Grouping \\
\hline MB-Clear & $-2.1(2.4)$ & $-0.5(0.6)$ & $6.2(0.7)$ & $6.9(1.3)$ & $\mathrm{B}$ \\
MB-Ivory & $-3.6(0.7)$ & $1.7(1.6)$ & $15.2(2.5)$ & $15.8(2.7)$ & $\mathrm{A}$ \\
SB-Clear & $0.3(1.2)$ & $-0.2(0.3)$ & $0.4(0.7)$ & $1.3(0.4)$ & $\mathrm{C}$ \\
SB-Ivory & $-1.2(0.7)$ & $-0.1(0.4)$ & $-1.1(0.9)$ & $1.8(1.0)$ & $\mathrm{C}$ \\
\hline
\end{tabular}

The s.d. values are shown in parentheses.

Grouping: Identical capital letters indicate that there are no statistical differences between the $\Delta \mathrm{E}^{*}$ values ( $>>0.05)$. 
tion of self-curable resin materials used with an amine reducing agent was caused by oxidation of the amine. Regarding the MB materials, the increase in $b^{*}$ value, which is considered to be a yellowish or brownish discoloration of the material, was apparent after aging compared to that of the $\mathrm{SB}$ materials (Table 2). The amine reducing agent contained in the $\mathrm{MB}$ materials was probably responsible for the increase in the $b^{*}$ value of the MB materials. Further, the conversion of the adhesives might have affected color stability in this study. Hirabayashi and Imai ${ }^{21)}$ reported that the amount of residual monomers of BPO-amine redox-initiated MMA-PMMA materials was greater than that of TBB-initiated MMA-PMMA materials.

As shown in Fig. 1 , the $\Delta E^{*}$ value of the $M B$ materials increased considerably 4 weeks or more after immersion in water. Unstable color change within 4 weeks is probably derived from both the ongoing polymerization reaction and material discoloration. The authors speculate that the reaction of the amine reducing agent with another acidic compound to form charge transfer complexes is one of the main factors for discoloration. Such a reaction tends to continue even after initiation and propagation reactions have settled down. Thus, the discoloration of the MB materials might have been worsened by the reaction between the carboxylic monomer and amine, both of which were included in the liquid of the MB material - which leads to the $\Delta \mathrm{E}^{*}$ value increasing proportionally after 4 weeks. It is also understandable that the slight color change of the SB materials was due to the absence of any amine reducing agent in the composition, although an aromatic carboxylic monomer (4-META) was added to the liquid component.

In addition to the influence of immersion period and material type, the difference in shade also affected color change. Although there were no significant differences between the $\Delta \mathrm{E}^{*}$ values after 24 weeks for the SB-Clear and SB-Ivory materials, that of the MB-Ivory material was significantly higher than that of the MB-Clear material. Though the reason for the difference was not proved in this study, the existence of different types of pigment and/or the different surface treatments of the pigments might be related to the color change.

Johnston and $\mathrm{Kao}^{22)}$ observed that the chromatic values were deemed to be slight if the $\Delta \mathrm{E}^{*}$ value was less than 1 and that values between 1 and 2 were clinically acceptable. Goldstein and Schmitt ${ }^{23)}$ reported that an $\Delta \mathrm{E}^{*}$ value of more than 3.7 was no longer within the limits of clinical acceptability. In the current study, it was considered that the color of the TBB-initiated acrylic adhesive material was more stable than that of the BPO-amine-initiated material, since an acceptable $\Delta \mathrm{E}^{*}$ value according to the definitions described above was not accomplished by the
MB material. When acrylic adhesive materials are applied for color adjustment of a restoration and/or a fixed splint, clinicians should consider their colorimetric stabilities and select the material accordingly, since the TBB-initiated acrylic adhesive material was proved to be superior colorimetrically to the BPO-amine initiated acrylic adhesive.

Color stability is a prerequisite for tooth-colored dental materials. While staining derived from material surface roughness, food, beverages, and cigarettes can be removed by means of a rotary instrument combined with a polishing agent, material discoloration is caused by the chemical ingredients added as part of the composition of the material. Clinicians cannot control color stability of any material serving in an oral cavity. Therefore, selection of materials with excellent color stability is very important regardless of the type or shape of the material or the location in which it will be applied.

\section{REFERENCES}

1) Touati B, Miara P. Light transmission in bonded ceramic restorations. J Esthet Dent 1993; 5: 11-18.

2) Vogel RI. The use of a self-polymerizing resin with enamel etchant for temporary stabilization. J Periodontol 1976; 47: 69-71.

3) Fusayama T. Adhesive resin splint. Oper Dent 1989; 14: 93-95.

4) Nakabayashi N, Kojima K, Masuhara E. The promotion of adhesion by infiltration of monomers into tooth substrates. J Biomed Mater Res 1982; 16: 265273.

5) Kimura M, Matsushige K, Kazama H. Development of new primer system containing a borate derivative. $J$ Dent Res 2002; 81(A) 75: \#399.

6) Koishi Y, Tanoue N, Atsuta M, Matsumura H. Influence of visible-light exposure on color stability of current dual-curable luting composites. J Oral Rehabil 2002; 29: 387-393.

7) May KB, Razzoog ME, Robinson E. Denture base resins: comparison study of color stability. J Prosthet Dent 1992; 68: 78-82.

8) Purnaveja S, Fletcher AM, Ritchie GM, Amin WM, Moradinas S, Dodd AW. Color stability of two selfcuring denture base materials. Biomater 1992; 3: 249250.

9) Buyukyilmaz S, Ruyter IE. Color stability of denture on base polymers. Int $\mathrm{J}$ Prosthodont 1994; 7: 372-382.

10) Noie F, O'Keefe KL, Powers JM. Color stability of resin cements after accelerated aging. Int $\mathrm{J}$ Prosthodont 1995; 8: 51-55.

11) Abu-Bakr N, Han L, Okamoto A, Iwaku M. Color stability of compomer after immersion in various media. J Esthet Dent 2000; 12: 258-263.

12) Swift EJ, Hammel SA, Lund PS. Colorimetric evaluation of vita shade resin composites. Int $\mathrm{J}$ Prostodont 1994; 7: 356-361.

13) Vichi A, Ferrari M, Davidson CL. Color and opacity 
variations in three different resin-based composite products after water aging. Dent Mater 2004; 20: 530534.

14) Asmussen E. An accelerated test for color stability of restorative resins. Acta Odontol Scand 1981; 39: 329332.

15) Ruyter IE, Nilner K, Moller B. Color stability of dental composite resin materials for crown and bridge veneers. Dent Mater 1987; 3: 246-251.

16) Brauer GM. Color changes of composites on exposure to various energy sources. Dent Mater 1988; 4: 55-59.

17) Peutzfeldt A, Asmussen E. Color stability of three composite resins used in the inlay/onlay technique. Scand J Dent Res 1990; 98: 257-260.

18) Geurtsen W. Substances released from dental resin composites and glass ionomer cements. Eur J Oral Sci
1998; 106(2 Pt 2): 687-695.

19) Ferracane JL. Elution of leachable components from composites. J Oral Rehabil 1994; 21: 441-452.

20) Asmussen E. Factors affecting the color stability of restorative resins. Acta Odontol Scand 1983; 41: 11-18.

21) Hirabayashi C, Imai Y. Studies on MMA-TBB resin. I. Comparison of TBB and other initiators in the polymerization of PMMA/MMA resin. Dent Mater J 2002; 21: $314-321$.

22) Johnston WM, Kao EC. Assessment of appearance match by visual observation and clinical colorimetry. $\mathrm{J}$ Dent Res 1989; 68: 819-822.

23) Goldstein GR, Schmitt GW. Repeatability of a specially designed intraoral colorimeter. J Prosthet Dent 1993; 69: $616-619$. 